\title{
QTL Mapping Integrated with BSA-Seq Analysis Identifies a Novel Gene Conferring Resistance to Brown Planthopper from Common Wild Rice (Oryza rufipogon Griff.)
}

Xuan Wang ( $\nabla$ wangxuan01234@126.com )

Guangxi University https://orcid.org/0000-0001-8692-8229

Yue Han

Guangxi University

Yuexiong Zhang

Guangxi Academy of Agricultural Science

Biao Deng

Guangxi Academy of Agricultural Science

\section{Biqiu Wu}

Guangxi Academy of Agricultural Science

\section{Xinying Guo}

Guangxi University

Yufen Qin

Guangxi University

\section{Yaoyu Fang}

Guangxi University

\section{Fang Liu}

Guangxi University

\section{Baoxiang Qin}

Guangxi University

Jijing Luo

Guangxi University

Rongbai Li

Guangxi University

\section{Research Article}

Keywords: brown planthopper, wild rice, resistance gene, BSA-seq, QTL analysis, marker-assisted selection 
Posted Date: May 11th, 2021

DOI: https://doi.org/10.21203/rs.3.rs-478718/v1

License: (9) This work is licensed under a Creative Commons Attribution 4.0 International License. Read Full License

Version of Record: A version of this preprint was published at Euphytica on February 26th, 2022. See the published version at https://doi.org/10.1007/s10681-021-02964-z. 


\section{Abstract}

The brown planthopper (Nilaparvata lugens Stål, BPH) is one of the most destructive rice pests worldwide. GXU202 is a germplasm of common wild rice (Oryza rufipogon Griff. ) with high resistance to the BPH. In this study, the genetic analysis indicated that the BPH resistant phenotype of GXU202 is controlled by a major gene. Through the combination and comparison of QTL linkage and BSA-seq analyses, a novel gene locus $B P H 41$ conferring $\mathrm{BPH}$ resistance was identified, which has been finely mapped to a 114-kb region delimited by D01031 and W1 on chromosome 4. The markers D01031 and D01045 showed high accuracy in predicting resistant phenotypes to BPH, suggesting their reliability for marker-assisted selection of $B P H 41$ in breeding for $\mathrm{BPH}$ resistant rice varieties. The present identification of $B P H 41$ will establish a foundation for further map-based cloning and functional characterization of the gene.

\section{Introduction}

Rice (Oryza sativa L.) is one of the most important staple food crops and plays an important role in agricultural production. Brown planthopper (BPH; Nilaparvata lugens Stål) is a devastating pest of rice prevalent across Asia (Normile 2008). They damage the rice plants by sucking the sap from phloem and also cause the infection of viruses, such as grassy stunt virus and ragged stunt virus(Zheng et al. 2020). Heavy BPH infestation leads to complete drying of rice plants and the occurrence of 'hopper burn' that causes severe yield losses during rice production(Backus et al. 2005). Over the long term, to reduce the application of pesticide that can result in environmental pollution, high cost, pest resurgence, and stronger pathogen strain virulence(Frisvold 2019), and subsequently utilizing BPH resistance genes to develop resistant cultivars is the most promising strategy in the management of BPH(Bisht et al. 2019). To this end, the exploitation of genes or genetic loci conferring BPH resistance from different germplasm resources could facilitate breeding programs for resistant rice varieties. Previous studies have indicated that the genetic basis of BPH resistance is complex, in which both major and minor resistance genes with complementary or additive effects, as well as environmental interactions are involved(Zheng et al. 2020).

To date, $40 \mathrm{BPH}$ resistance genes have been identified from cultivated and wild species of rice(Akanksha et al. 2019). Among them, at least thirteen genes are clustered in two distinct regions of chromosome 4(Hu et al. 2016). This phenomenon is consistent with the pattern of $\mathrm{R}$ genes, which are usually distributed on chromosomes in clusters(Mizuno et al. 2020). Specifically, five of them (Bph6, bph16, bph18, BPH27, Bph27(t) and Bph34) distribute on the long arm of chromosome 4(Guo et al. 2018; Hirabayashi et al. 1998; Fujita et al. 2013; Huang et al. 2013; He et al. 2013; Kumar et al. 2018); as well, eight genes (Bph3, Bph12, Bph15, Bph17, Bph20, Bph30, Bph33, and BPH36) and four QTLs (QBph4, QBph4.2, QBph4.3 and QBph4.4) distribute on the short arm of chromosome 4(Liu et al. 2015; Qiu et al. 2012; Lv et al. 2014; Sun et al. 2005; Rahman et al. 2009; Wang et al. 2018; Hu et al. 2018; Li et al. 2019; Hu et al. 2015; Kamolsukyeunyong et al. 2019; Mohanty et al. 2017). 
Wild rice species have abundant genetic diversity for studying the genetic basis of resistance phenotype to $\mathrm{BPH}(\mathrm{Hu}$ et al. 2016). Previous studies have identified $24 \mathrm{BPH}$ resistance genes/QTLs in 7 wild rice species with different genome types. Of which, bph2O(t), bph21(t), bph22(t), bph23(t), Bph27, Bph29, $B P H 35$ and $B P H 36$ were derived from O. rufipogon with the AA genome(Yang et al. 2012; Hou et al. 2011; Huang et al. 2013; Wang et al. 2015; ZHANG et al. 2020; Li et al. 2019); Bph34, bph39, and bph40 were derived from O. nivara with the AA genome(Kumar et al. 2018; Akanksha et al. 2019); bph11, bph16, Bph14, Bph15, and QBph4.1 were iderived from O. officinalis with the CC genome(Sharma et al. 2003; Hirabayashi et al. 1998; Du et al. 2009; Lv et al. 2014; Hu et al. 2015); Bph13 derived from O. eichingeri or 0 . officinalis in two different research(Liu et al. 2001; Renganayaki et al. 2002); Bph12 was derived from O. latifolia with the CCDD genome(Qiu et al. 2012); Bph20 and Bph21 were derived from O. minuta with the CCDD genome(Rahman et al. 2009); Bph10, Bph18, and QBph4.2 were derived from O. australiensis with the EE genome(Ishii et al. 1994; Ji et al. 2016; Hu et al. 2015). Therefore, identifying more BPHresistance genes/QTLs from wild rice species is of great significance in rice breeding practice.

QTL mapping is a fundamental approach for understanding the genetic inheritance of phenotypic variation. It is wildly utilized in map-based cloning of candidate genes for QTLs and can provide valuable markers for marker-assisted selection (MAS) in crop breeding(Gupta et al. 2019). This conventional approach involves in developing the genetic population with segregating phenotype of target traits and requires to identify hundreds of polymorphic markers to genotype a large number of individuals derived from the biparental cross. Thus, it is a time-consuming and laborious process and therefore has become a bottleneck for traditional QTL mapping studies. The combination of bulked segregant analysis with whole-genome sequencing (BSA-seq) can used to facilitate the mapping of qualitative trait that is controlled by a major gene, and has been successfully applied for the rapid identification of important agronomical traits in many crop plants(Nguyen et al. 2019), such as rice, maize, wheat, barley, soybean, potato, groundnut, and sorghum(Abe et al. 2012; Klein et al. 2018; Navarro-Escalante et al. 2020; Jia et al. 2017; Song et al. 2017; Kaminski et al. 2016; Pandey et al. 2017; Han et al. 2015).

In rice, BSA-seq has been successfully applied for identifying genes conferring resistance to biotic and abiotic stresses. Wu et al. (2019) mapped the genes related with the partial blast resistance between 1.52 and 4.32 Mb on chromosome 6. Liang et al.(2020) identified a pi21 locus conferring basal blast resistance between $18.90-22.10 \mathrm{Mb}$ on chromosome 4 . Sun et al. (2018) identified a cold-tolerant locus between 21.84-23.65 Mb on chromosome 6. Tao et al. (2018) identified a aluminum toxicity tolerance gene between $4.72-4.87 \mathrm{Mb}$ on chromosome 2.Therefore, it is also a promising approach for the identification of $\mathrm{BPH}$ resistance gene.

In the present study, we focused on the identification of the gene conferring BPH resistance in a common wild rice germplasm GXU202 through a combination of conventional QTL mapping and the BSA-seq approach via linkage analysis. This study aimed to (1) discover new resistance gene loci associated with $\mathrm{BPH}$ resistance using BSA strategy; (2) validate the loci through conventional QTL mapping and perform comparison between both methods to evaluate their reliability; (3) propose the candidate genes based on 
the genome annotation; and (4) develop reliable markers to facilitate MAS in BPH-resistance breeding programs.

\section{Materials And Methods}

\subsection{Plant materials, mapping population, and backcrossing generations development}

GXU202 (accession number) is a germplasm of common wild rice (O. rufipogon Griff.) collected from Guangxi Province, China, and maintained in Guangxi University with high resistance to BPH infestation. 9311 is an indica rice variety that is widely cultivated in Southern China and that is highly susceptible to BPH. GXU202 was crossed with 9311, and the resulting $F_{1}$ individuals were self-crossed twice to obtain the $F_{2}$ individuals and $F_{2: 3}$ lines that were used as materials for screening $B P H$ resistance lines. The obtained resistance lines were subsequently used for QTL mapping and BSA sequencing analysis. The highly resistant- $F_{3}$ individuals (score 0-3) were selected to be continuously backcrossed with 9311 to generate $\mathrm{BC}_{2} \mathrm{~F}_{1}$ populations. The $\mathrm{BC}_{2} \mathrm{~F}_{1}$ individuals were self-fertilized twice to obtain $\mathrm{BC}_{2} \mathrm{~F}_{2: 3}$ lines, which were selected for phenotypic evaluation and genotyping analysis to verify of the accuracy of the mapping. All the plants were grown in the experimental paddy field (Nanning, $22.85^{\circ} \mathrm{N}, 108.26^{\circ} \mathrm{E}$ ) located in Guangxi University, Nanning, Guangxi, China.

\subsection{Evaluation of $\mathrm{BPH}$ resistance}

The BPH insects were captured from rice field in Nanning, Guangxi province, China, and reared on the susceptible indica rice variety Taichung Native 1 (TN1) plants to produce enough nymphs for infestation in cages $(50 \times 50 \times 100 \mathrm{~cm})$ surrounded with light-transmitting fine nylon mesh. The cages and seedlings were maintained in a greenhouse under natural daylight at $26-32^{\circ} \mathrm{C}$ condition. The standard seedbox screening test (SSST) was performed on the $\mathrm{F}_{2: 3}$ families to evaluate the level of $\mathrm{BPH}$ resistance. Each mesh-covered tray $(58 \times 38 \times 9 \mathrm{~cm})$ filled with field mud $(\sim 5 \mathrm{~cm}$ deep) was averagely planted 11 rows, including two rows of the resistant (GXU202) and susceptible (9311) parental lines. Each row was planted 20-25 plants of one family. At third-leaf stage (approximately 15 days after sowing), the seedlings were infested with second- or third-instar BPH nymphs at an average level of five insects per seedling and covered with light-transmitting fine nylon-net mesh enclosure $(52 \times 32 \times 45 \mathrm{~cm})$. When all of susceptible control seedlings were wilted (scored as 9), the BPH resistance of each individual seedling was assessed according to the rating scale proposed by the International Rice Research Institute (IRRI 1988) (0, no damage; 1 , very slight damage; 3 , first and second leaves partially yellowing; $5=$ pronounced yellowing and stunting; 7 = mostly wilting, the plant still alive; $9=$ the plant completely wilted or died).

The resistance score of each $\mathrm{F}_{2}$ individual was inferred from the average resistance score of the seedlings in the corresponding $\mathrm{F}_{2: 3}$ families. The test was conducted three times, and the average resistance score of each $\mathrm{F}_{2}$ lines was used in subsequent analysis.

\subsection{Sample Collection, Construction of Mixed Pools and DNA Extraction}


Fresh leaves of "GXU202", "9311", and the $F_{2}$ individuals were collected at the tillering stage. Genomic DNA was extracted from the leaves for genotyping and QTL analysis. The pools were generated by respectively mixing equal amounts of DNA of 30 extreme resistant (resistance score 0 4.0) $F_{2}$ individuals and of 30 extreme susceptible (resistance score 8.0 9.0) $F_{2}$ individuals for library construction and whole genome sequencing. The total genomic DNA was isolated from the leaf tissues using the cetyltrimethylammonium bromide (CTAB) method(Murray and Thompson 1980). The quality and quantity of isolated DNA were determined using a NanoDrop 2000 Spectrophotometer (Thermo Scientific, Wilmington, $\mathrm{DE}, \mathrm{USA}$ ), and the final concentration was adjusted to $80 \mathrm{ng} \bullet \mu \mathrm{L}^{-1}$.

\subsection{Whole Genome Resequencing and Data processing}

DNA samples of the two parents and the two pools, were prepared for library construction, which were named R01 (GXU202), R02 (9311), R03 (resistant pool) and R04 (susceptible pool), respectively. After quality inspection, DNAs of four samples (R01, R02, R03, and R04) were randomly broken into 350-bp fragments by ultrasonication; the fragments were then subjected to end repair, addition of nucleotide (A) overhangs, ligation with sequencing adapters, and amplification by PCR. The purified products were used to construct a library for whole genome resequencing using the Illumina HiSeq platform. Whole genome resequencing was conducted by Biomarker Technologies (Beijing, China).

To get high-quality reads for subsequent information analysis, the raw data (raw reads) of fasta format was first processed through a series of quality control (QC) procedures to remove low quality paired reads. QC standards were carried out as follows: (1) removing the reads with adapter; (2) removing pair end reads with $\geq 10 \%$ unidentified nucleotides $(N)$; (3) removing reads with $>50 \%$ bases having Qphred quality $<10$. Clean reads of each sample were aligned to the reference genome of $O$. sativa $L$. ssp. indica cv. 9311 (ftp://ftp.ensemblgenomes.org/pub/plants/release-45/fasta/oryza_indica/dna/) using the Burrows-Wheeler Aligner (BWA) software(Li and Durbin 2009). Based on the mapping results of the clean reads, the tool of Mark Duplicate in Picard (https://sourceforge.net/projects/ picard/) was used to eliminate PCR duplication. After pretreatment of local realignment and base recalibrationSinglenucleotide polymorphism (SNP) and Small InDel calling was performed by using the Genome Analysis Toolkit (GATK) software to increase SNP/Small InDel calling accuracy(McKenna et al. 2010).

\subsection{Association analysis of SNPs and Indels}

To identify candidate genomic region responsible for BPH resistance, the association analysis of SNPS was conducted by Euclidean Distance (ED) and SNP-index algorithm, respectively(Hill et al. 2013). Before the association analysis, to obtain high quality and reliable SNP sites, SNPs were excluded from the two bulk sequences according to the following criteria: (1) with multiple genotypes; (2) read depth < 4; (3) genotypes were the same between the pools; and (4) the dominant mixed pool gene does not come from dominant parents.

The ED between the allele frequencies at each SNP site in the R- and S-pools was calculated using the following equation: 
Each letter $(A, C, G, T)$ indicates the frequency of the corresponding DNA nucleotide. $X_{\text {mut }}$ and $X_{W T}$ represent the frequency of $X$ base in mutant and wild type bulk, respectively. To eliminate the background noise, the fifth power of the original ED value was regarded as a correlation value. The correlation threshold for the significance of marker-trait associations was set at median + 3SD of the DISTANCEfitted values. The genome regions at which the DISTANCE-fitted values exceeded the threshold were designated as candidate regions associated with resistance to $\mathrm{BPH}$.

Another association analysis was performed using SNP-index method, which is indicated by $\Delta$ (SNPindex) through finding significant differences in genotype frequency between the R- and S-pools. SNPindex and delta SNP-index were performed using the method based on the depth information generated in variants calling. The $\Delta$ (SNP-index) was calculated as follows: $\Delta$ (SNP-index) $=$ SNP-index (extreme resistant) - SNP-index (extreme susceptible)= Maa/(Maa+Paa) $-\mathrm{Mab} /(\mathrm{Mab}+\mathrm{Pab})$, where Maa indicates the depth of the aa population derived from 9311; Paa indicates the depth of the aa population derived from GXU202; Mab indicates the depth of the ab population derived from 9311; Pab indicates the depth of the ab population derived from GXU202. In order to eliminate false positive sites, the $\Delta$ (SNP-index) data were of the SNPs on the same chromosome fitted using DISTANCE method(Fekih et al. 2013). The stronger the correlation between SNP and traits, the closer the $\triangle$ (SNP-index) value is to 1, and SNPs with $\triangle$ (SNP-index) higher than the threshold were regarded as a target region that might be underlying BPH resistance of rice. ED and $\triangle S N P$-index values were plotted, and the intersections between candidate regions identified using the two methods were designated as candidate regions associated with BPH resistance.

The association analysis of small InDels was also conducted by Euclidean Distance (ED) and InDel-index algorithm, respectively, following the same method as that of SNPs(Singh et al. 2017). Finally, the overlapping regions of the candidate regions based on SNP and indel association analysis, were designated as the final candidate intervals.

\subsection{Genotyping, Genetic Map Construction, and QTL Analysis}

According to the result of preliminary mapping identified through BSA-seq, we chose chromosome 4 to develop the genetic linkage map using GXU202/9311 $F_{2}$ population. A total of 310 simple sequence repeat (SSR) markers and the insertion/deletion (InDel) markers distributed across chromosome 4 of the rice genome were obtained from GRAMENE database (https://archive.gramene.org/markers/microsat/). The molecular markers were initially screened for polymorphism between GXU202 and 9311 for QTL mapping analysis to detect the gene locus for BPH in GXU202. The sequences of the polymorphic markers are listed in Table S9.

The selected polymorphic markers were used to genotype the 255 individuals of the $F_{2}$ population. QTL analysis was performed with the inclusive composite interval mapping (ICIM) using the BIP (QTL 
mapping in the biparental populations) functionality of QTL IciMapping 4.1(Meng et al.

2015). LOD thresholds were calculated with 1,000 permutation tests $(P<0.05)$ and used to declare a putative QTL.

PCR reactions were in a final volume of $10 \mu \mathrm{L}$ with PCR mixture included $1 \mu \mathrm{L}$ template DNA (60 $\mathrm{ng} \cdot \mu \mathrm{L} 1)$, $5 \mu \mathrm{L} 2 \times$ Taq Master Mix, $0.5 \mu \mathrm{L}$ each of the forward and reverse primers $(10 \mu \mathrm{M})$, and $3 \mu \mathrm{L} \mathrm{ddH}_{2} \mathrm{O}$. The PCR was performed in a $1100^{\mathrm{TM}}$ thermal cycler (Bio-Rad, USA) as following program: initial denaturation at $95^{\circ} \mathrm{C}$ for $5 \mathrm{~min} ; 35$ cycles of denaturation at $95^{\circ} \mathrm{C}$ for $30 \mathrm{~s}$, annealing at $56-62^{\circ} \mathrm{C}$ (for different markers) for $30 \mathrm{~s}$ and extension at $72^{\circ} \mathrm{C}$ for $30 \mathrm{~s}$; and a final extension step at $72^{\circ} \mathrm{C}$ for $5 \mathrm{~min}$. The amplified products were detected by agarose gel electrophoresis with a concentration of $1.5 \%$ or polyacrylamide gel electrophoresis (PAGE) with a concentration of $8 \%$.

\section{Results}

\subsection{Phenotyping of BPH resistance}

In our previous evaluations, the common wild rice germplasm GXU202 with high resistance to BPH was observed under natural nursery conditions (data not shown). According to the identification criteria of $\mathrm{BPH}$ in the seedling bulk test, we evaluated the resistance potential of GXU202, 9311, and $F_{2}$ populations derived from the cross GXU202/9311. After infestation for 15 days, GXU202 exhibited high resistance to the BPH insects, which was scored to be level 2.5 (Fig. 1a,b). In contrast, rice variety 9311 was highly susceptible to BPH, which was scored to be a level of 9.0 (Fig. 1a,b). These results indicated that GXU202 possesses high resistance to $\mathrm{BPH}$. A total of $255 \mathrm{~F}_{2: 3}$ families of $\mathrm{GXU} 202 \times 9311$ were infested with $\mathrm{BPH}$ and scored at $15 \mathrm{dpi}$. In the $\mathrm{F}_{2}$ population, $\mathrm{BPH}$-resistance scores showed a continuous range from 2.5 to 9.0, with a valley between 7.0 and 7.9 in the distribution curve (Fig. 1d). Such a distribution indicates the involvement of a major gene and minor QTLs in the segregation of BPH resistance in this population.

Based on the $\mathrm{BPH}$ resistance phenotypic scoring, the $255 \mathrm{~F}_{2}$ individuals could be grouped as: 62 individuals with score 0-4.9 were regarded as resistant groups, 128 individuals with score 5-7.9 were moderate susceptible groups, and 65 individuals with score 8-9 were classified to highly susceptible groups. The $\mathrm{F}_{2}$ populations with the phenotype of resistant, moderate susceptible, and highly susceptible presented a segregation ratio of $62: 128: 65$, which fits well with a 1:2:1 segregation model

$$
\left(\chi_{\mathrm{c}}^{2}=0.066<\chi_{0.0 \leq 2}^{2}=5.99, P>0.05\right) .
$$

The result indicated that the inheritance of BPH resistance in the study is affect by a single Mendelian gene that is derived from wild rice GXU202. It was tentatively designated as BPH41. For the BSA-seq analysis, 30 extremely resistant $F_{2}$ lines (resistance score 2.5-4.0) and 30 extremely susceptible $F_{2}$ lines (resistance score 8.9-9.0) were selected and bulked as the R pool and S pool, respectively.

\subsection{DNA Resequencing for BSA analysis}


The whole genome resequencing data were generated from resistant parents (GXU202, abbreviate as R01), susceptible parent (9311, abbreviate as R02), the extreme resistant bulk (abbreviate as R03) and the extreme susceptible bulk (abbreviate as R04) (Table 1). A total of $63.85 \mathrm{G}$ bp raw data was generated and were finally obtained a $63.25 \mathrm{G}$ bp of clean data after quality control and data filtering. The clean reads of each sample were 54.86 million for R01, 58.42 million for R02, 40.74 million for R03, and 57.07 million for R04, with high quality $(90.88 \%<$ Q30 < $91.62 \%)$ and stable GC content $(42.74 \%<$ GC $<45.44 \%)$ (Table 1). The high-quality clean reads were aligned to the genome of 9311 . There were $99.83 \%, 99.76 \%, 99.83 \%$, and $99.95 \%$ reads for R01, R02, R03, and R04 that could be properly mapped to $88.33 \%, 95.55 \%, 88.3 \%$, and $45.93 \%$ of the reference genomic sequence, respectively (Table 1 ). Unfortunately, the mapping rate of susceptible pool (R04) is abnormally low, which may be attributed to the sampling strategy. The sampling timepoint coincided with the outbreak of planthoppers in the field, the $F_{2}$ individuals proved to be highly susceptible in later experiment were inevitably infested by BPH and contaminated by microbes, which might be a possible reason that led to the low mapping rate of susceptible pool. However, the data can still be reliable for subsequent analysis because of the following reasons. The average genome coverage depth of the mapped reads of R01, R02, R03, and R04 samples are 35-, 41-, 26-, and 18-fold, respectively (Table 2). More than ten high-quality clean reads from R01, R02, R03, and R04 samples can cover $85.34 \%$, $88.78 \%, 88.1 \%$, and $83.27 \%$ of reference bases, respectively(Table 2, Fig. S1, S2). The SNPs and InDels were sufficiently and uniformly distributed throughout the whole genome, and the coverage depth was evenly distributed on chromosomes, indicating the fine sequencing randomness (Table 2, Fig. 2, Fig. S2). For R04, the percentage of reference bases with a coverage depth of 1 fold, 5 folds, and 10 folds reached $96.76 \%, 91.6 \%$, and $83.27 \%$, respectively(Table 2 ). As a result, these data can be used for subsequent SNP/InDel detection and analysis.

Table 1. Summary of data generated by sequencing and reads mapped to reference genome.

Sample Raw Reads Clean High-Quality Reads

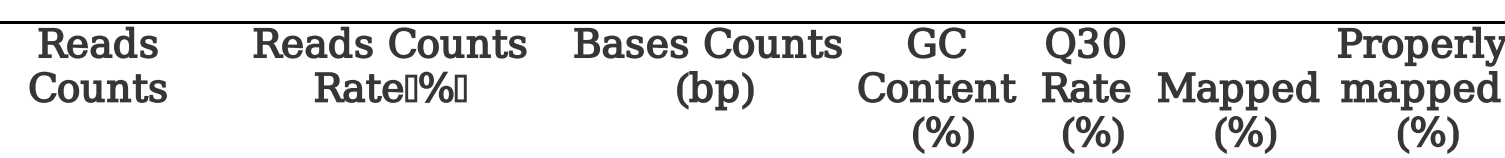

\begin{tabular}{cccccccc}
\hline R01 & $54,951,13054,859,002$ & 99.83 & 16435059806 & 43.64 & 91.62 & 96.76 & 88.33 \\
R02 & $58,565,96158,423,701$ & 99.76 & 17505340320 & 42.74 & 91.33 & 98.35 & 95.55 \\
\hline R03 & $40,806,33940,738,099$ & 99.83 & 12204672398 & 43.78 & 91.27 & 93.69 & 88.3 \\
\hline R04 & $57,096,29557,065,729$ & 99.95 & 17100679492 & 45.44 & 90.88 & 48.35 & 45.93 \\
\hline Total & 211419725211086531 & 99.84 & 63245752016 & & & & \\
\hline
\end{tabular}

Table 2. Summary of coverage depth and coverage ratio with different depth of the 9311 genome by the mapped reads 


\begin{tabular}{|c|c|c|c|c|}
\hline Sampl & age & Percentage of $\mathrm{R}$ & nce Bases with & rent Coverage $\mathrm{D}$ \\
\hline & & $\overline{\text { Coverage } \geq 1 \mathrm{X}(\%)}$ & Coverage $\geq 5 X(\%)$ & Coverage $\geq 10 \mathrm{X}(\%)$ \\
\hline R01 & 35 & 92.17 & 88.6 & 85.34 \\
\hline R02 & 41 & 92.67 & 90.32 & 88.78 \\
\hline R03 & 26 & 96.52 & 92.72 & 88.1 \\
\hline R04 & 18 & 96.76 & 91.6 & 83.27 \\
\hline
\end{tabular}

\subsection{SNP and Short InDel Polymorphism Profiling and Identification of Candidate Region for BPH resistance}

Based on the uniquely mapped reads, SNPs and indels between samples and reference were called and identified. In total, 2,440,152 SNPs, including 91,582 nonsynonymous coding SNPs, and 512,846 small InDels were detected on 12 chromosomes between the parental lines (R01 vs. R02). Between the two extreme bulks (R03 vs. R04), we detected 812,866 SNPs, including 28,304 nonsynonymous coding SNPs, and 171,431 small InDels (Table S1, 2; Fig. S3). The Venn diagrams indicated the relationships among R01, R02, R03, and R04 based on the identified SNPs and InDels (Fig. S3), in which the four samples shared 107278 SNP and 55337 InDel loci, respectively.

Before the association analysis, the SNP and InDel were filtered and resulted in 749,031 SNP sites and 137,651 InDel sites high-quality and credible data,. To determine the candidate regions for BPH resistance, the correlation value was calculated and plotted using the Euclidean distance (ED) algorithm and SNP/InDel-index algorithm. As expected, the plot of ED value of SNPs and small InDels showed an obvious peak at the same interval on chromosome 4, respectively. Therefore the candidate regions were detected, within which the SNP/InDel sites have higher ED value than the threshold (Fig. 2a, b; Fig. S4). For the SNP/InDel-index method, the candidate regions detected according to higher $\Delta$ (SNP-/InDel index) value than the threshold (confidence interval $>99 \%$ ). The detected candidate regions were mapped on the same range that was detected by ED algorithm (Fig. 3c,d; Fig. S4).

In detail, according to polymorphic SNPs, one candidate region covering $11.47 \mathrm{Mb}$ on chromosome 4 were obtained based on the threshold value of 0.32 using ED algorithm (Fig. 3a; Table S4), and four candidate regions on chromosome 4 were detected based on the $\triangle$ (SNP-index) value at a confidence interval higher than 0.99 (Fig. 3c; Table S4). Four candidate regions were overlapped based on the results of the two analysis methods. The regions were located on chromosome 4 ranged from $4.51 \mathrm{cM}$ to $7.54 \mathrm{cM}$, covering a total length of $1.71 \mathrm{Mb}$ with 98 annotated genes (Table S5).

The same methods were used to analyze the polymorphic small InDels. Similarly, one candidate region covering $11.44 \mathrm{Mb}$ on chromosome 4 was obtained based on the threshold value of 0.33 using ED algorithm (Fig. 3b; Table S6), and five candidate regions on chromosome 4 were obtained based on the $\Delta$ (InDel-index) value at a confidence interval higher than 0.99 (Fig. 3d; Table S7). Five candidate regions 
were overlapped based on the results of the two analysis methods. The regions were located on chromosome 4 ranged from $3.52 \mathrm{cM}$ to $8.40 \mathrm{cM}$, covering a total length of $3.70 \mathrm{Mb}$ with 224 annotated genes (Table S8).

Finally, taking overlapping targeted regions from the SNP and InDel association analysis into account, 4 tightly successive regions spanning 4.51 7.54 Mb on chromosome 4 for BPH resistance were identified. A total length of $1.71-\mathrm{Mb}$ region with 98 annotated genes was regarded as a candidate region for the BPH41 locus (Table 3 ).

Table 3. The detailed positions of the candidate regions for BPH resistance based on the ED and SNP/InDel index analysis.

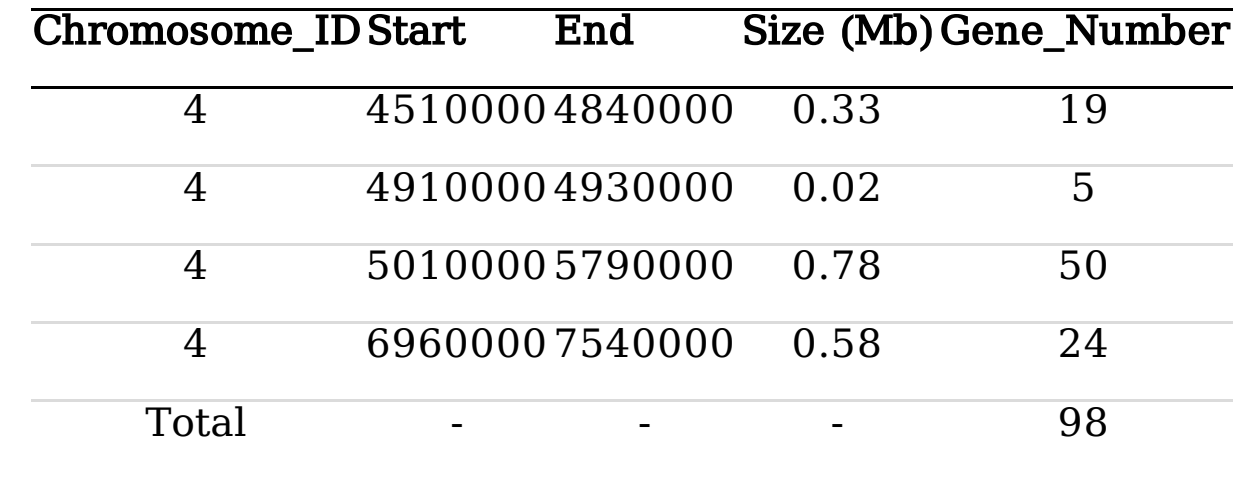

\subsection{Confirmation of the detected Regions by Conventional genetic Mapping of chromosome 4}

To validate the candidate region identified by BSA-seq, a genetic linkage map of chromosome 4 was constructed. A total of 310 SSR markers distributed across chromosome 4 were used to assess the polymorphism between the resistant parent (GXU202) and susceptible parent (9311). As a result, 23 polymorphic SSR markers evenly distributed on chromosome 4 were selected. The primer sequences for these markers are shown in Table S9. They were used to genotype the total 255 individuals of the $F_{2}$ population for QTL analysis. Then, a genetic map spanning $34.58 \mathrm{cM}$ of chromosome 4 was generated using QTL IciMapping 4.0 to identify BPH resistance QTL(s) based on resistance score (Fig. 1d). The average marker interval of the genetic map was $1.50 \mathrm{cM}$, and the maximum distance of interval is 2.49 $\mathrm{cM}$ (Table S9). The order of the markers on the map is consistent with the physical order of the markers on chromosome 4 of 9311 . As expected, a significant QTL peak was detected at $5.25 \mathrm{cM}$ of chromosome 4, which is located in the interval of marker D01031(4.233Mb) and D01045(5.384Mb) (Fig. 3a). The locus had a highest LOD score of 15.10 and accounted for $25.41 \%$ of the phenotypic variation of BPH resistance, which is consistent with the results identified by BSA sequencing. Thus, it is considered more likely to harbour the $\mathrm{BPH} 41$ locus than other regions.

Another QTL was detected at $11.25 \mathrm{cM}$ of chromosome 4 flanked by marker D01079 and D01089 explained the phenotypic variance were 7.43 (Fig. 3a), which might had minor effect in the response to $\mathrm{BPH}$.

\subsection{Fine mapping of BPH41}


The preliminary mapping region of $B P H 41$ partially overlapped with another $\mathrm{BPH}$ resistance gene $B P H 12$, which located in an interval spanning 5.21-5.66 cM on the short arm of chromosome 4(Qiu et al. 2012). To further identify the exact location of $B P H 41$, the genotypes of randomly chosed $541 \mathrm{BC}_{2} \mathrm{~F}_{2}$ seedlings developped from the cross of GXU202 and 9311 were screened using SSR markers D01031 and D01045, resulting in the identification of 9 recombinants. Besides, more SSR markers were developed in the interval flanked by D01031 and D01045, and three polymorphic markers were obtained, named W1, W2 and W3. Together with D01031 and D01045, these five markers were surveyed to assay the genotype of the 9 recombinants for fine mapping of $B P H 41$.

At the same time, the 9 recombinants and the two parental lines were also surveyed in the seedling bulk test for resistance to BPH insects. Lines L1 and L2 were homozygous for 9311 alleles at D01031 and W2, which were confirmed to be susceptible to BPH. In contrast, Lines L8 and L9 were homozygous for GXU202 alleles at D01031 and W2, which showed a high level of resistance to the BPH, with an average resistance score of 4.9 and 4.6, respectively. Lines L3, L4, L5, L6 and L7 were heterozygous at D01031 and W2, which had moderate resistance to the BPH and scored 7.4, 7.1, 7.0, 6.7 and 6.4, respectively(Fig. $3 \mathrm{~b}, \mathrm{c})$. Thus, based on the analysis of the genotypes and resistance scores of the 9 recombinants, $B P H 41$ locus was localized in the region delimited by D01031 and W2(Fig. 3c,d).

According to the primer sequences information, the physical distance between D01031 and W2 is estimated to be 114-kb in 9311 genome according to the database of eRice (http://www.elabcaas.cn/rice/genome_9311.html).

\subsection{Validation of SSR marker for BPH resistance selection}

In the QTL analysis, the locus with the highest LOD score was flanked by markers D01031 and D01045 (Table 5; Fig. 4). In parallel with screening for recombinants, the total of $541 \mathrm{BC}_{2} \mathrm{~F}_{2}$ individuals derived from the cross GXU202 $\times 9311$ were also utilized for validation of the efficacy of D01031 and D01045, in order to develop effective molecular markers for BPH resistance selection. This was performed by genotyping the $\mathrm{BC}_{2} \mathrm{~F}_{2}$ population and the individuals homozygous for the resistant allele were selected to evaluated for $\mathrm{BPH}$ resistance using their corresponding $\mathrm{BC}_{2} \mathrm{~F}_{2: 3}$ lines. For both markers, $\mathrm{PCR}$ amplification yielded clear bands for two parents, homozygous, and heterozygous individuals from the segregating populations(Fig. S5). As expected, both markers showed high co-segregation with the BPH reaction. There were 80 homozygous $\mathrm{BC}_{2} \mathrm{~F}_{2}$ plants among the $541 \mathrm{BC}_{2} \mathrm{~F}_{2}$ individuals, including 76 individuals homozygous at both site and 4 recombinants (lines L3, L5, L8 and L9). Except for lines L3 and L5, which were heterozygous at D01031, all the other individuals showed a resistance score of 0-6.9, which significantly improved compared with the backcross parents 9311 . Thus, the accuracy of D01031 and D01045 was $100 \%$ and $97.44 \%$ in BPH resistance selection, respectively, and can be used for introgressing BPH41 in the background of 9311.

\section{Discussion}




\subsection{The integration of BSA-seq and QTL analysis identified a gene locus on Chromosome 4 of GXU202}

In rice, the damage caused by $\mathrm{BPH}$ is a restriction factor for production. Genetic analysis of BPH resistance and the identification of resistance genes are important for pest control and prevention. Common wild rice GXU202 is a highly resistant germplasm to BPH, while indica cultivated rice variety 9311 exhibited obvious susceptibility (Fig. 1). In the current study, a total of $255 \mathrm{~F}_{2: 3}$ lines from a cross between GXU202 and 9311 were constructed to identify the gene locus for BPH resistance. The segregation ratio of resistant to susceptible individuals (1:3) in $\mathrm{F}_{2}$ populations fitted Mendel's law of segregation, indicating that $\mathrm{BPH}$ resistance trait in GXU202 was regulated by a major gene.

Based on a monogenic inheritance hypothesis, we combined the BSA-seq and QTL analysis to identify the gene for BPH resistance in GXU202. Our study revealed a key genetic locus, designated as $B P H 41$ for $\mathrm{BPH}$ resistance at the seedling stage of rice. Through the combination of BSA-seq and QTL analysis, $B P H 41$ was primarily mapped around at $5.25 \mathrm{cM}$ on the short arm of chromosome 4 in the interval delimiting by the markers $\mathrm{D} 01031(4.233 \mathrm{Mb})$ and $\mathrm{D} 01045(5.384 \mathrm{Mb})$ from an F2 population. In the further fine mapping using BC2F2 population, its position finally delimited to a region flanked by the markers D01031 and W1, which are approximately $114 \mathrm{~kb}$ apart in the 9311 genome.

\subsection{BPH41 located in a region clustering several BPH resistance genes}

To date, $40 \mathrm{BPH}$ resistance genes have been mapped on rice genome. It has been noted that these BPHresistance genes appear to be clustered on rice chromosomes 3, 4, 6, and 12(Hu et al. 2016). At least 8 genes have been mapped to the adjacent or overlapped regions on the short arm of chromosome 4, including BPH3, BPH12, Bph15, Bph17, Bph20(t), Bph30, Bph33 and Bph36(Liu et al. 2015; Qiu et al. 2012; Lv et al. 2014; Sun et al. 2005; Rahman et al. 2009; Wang et al. 2018; Hu et al. 2018; Li et al. 2019). Bph41 also occupies this cluster, and roughly 3.3 Mb downstream from the $B p h 33$, and 1.0 Mb upstream from Bph12. The location of BPH-resistance genetic loci (genes and QTLs) on chromosome 4 supports the conjecture that the tightly linked loci tend to be present in cluster in the particular regions of chromosomes, and act in tandem( Mizuno et al. 2020).

\subsection{D01031 and D01045 are valuable molecular markers in MAS of BPH41 in breeding programs}

In the past few decades, molecular markers have played an important role in target gene location and marker-assisted selection (MAS) breeding. Identifcation of a tightly linked DNA marker is a prerequisite for rapidly identify the introgressions of the desired genetic locus. In the result of QTL analysis, two SSR molecular markers, D01031 and D01045, were tightly linked to the traits. In this study,D01031 and D01045 showed $100 \%$ and $97.44 \%$ accuracy in predicting resistance phenotypes to $\mathrm{BPH}$, rescepctively, proving their potential application the application of in breeding practice like marker-assisted selection and $\mathrm{BPH}$ resistant loci pyramiding for developing of rice cultivars with high $\mathrm{BPH}$ resistance.

It is worth noting that, compared to the high resistance of their donor parents in $\mathrm{F}_{3}$ generation (sored 1 or 3 ), the resistance of the backcross progenies was remaining, but declined. It could be considered that the 
existing of $B P H 41$ can indeed enhance the resistance level of plants, but stronger resistance seem to be the result of the complex interaction and synergistic effect of BPH41 and other minor QTLs harbored in GXU202.

\subsection{Further research prospective}

As a close ancestor of cultivated rice, wild rice species are rich sources of resistance genes against biotic and abiotic stress, such as cold, pest, pathogen, waterlogging, drought, salt and weed. Previous genetic studies have identified $24 \mathrm{BPH}$ resistance genes/QTLs in 7 wild rice species(Yang et al. 2012; Hou et al. 2011; Huang et al. 2013; Wang et al. 2015; ZHANG et al. 2020; Li et al. 2019; Kumar et al. 2018; Akanksha et al. 2019; Sharma et al. 2003; Hirabayashi et al. 1998; Du et al. 2009; Lv et al. 2014; Hu et al. 2015; Liu et al. 2001; Renganayaki et al. 2002; Qiu et al. 2012; Rahman et al. 2009; Ishii et al. 1994; Ji et al. 2016; Hu et al. 2015). These genes have an increasingly wide utilization in the breeding work, in order to improve the $\mathrm{BPH}$ resistance of the cultivated rice varieties. In this study, $B P H 41$ was detected in wild rice $O$. rufipogon. The introgression of the fragment containing $B P H 41$ into indica rice variety 9311 enhanced the $\mathrm{BPH}$ resistance.

However, the population carrying the introgression fragments of wild rice species have complex genetic background. Therefore, in further study of $B P H 41$, more advanced backcrossing lines like recombinant inbred lines (RILs) and near isogenic lines (NILs) were required to be constructed and applied to further fine mapping and cloning the gene underlying $\mathrm{BPH}$ resistance. On one hand, fine mapping of $\mathrm{BPH} 41$ with high resolution can be achieved using these rice lines. On the other hand, these advanced backcrossing lines are valuable intermediate material in BPH resistance breeding.

The findings in this study will facilitate further exploration of $B P H 41$ locus, such as map-based cloning and, transformation experiments of candidate genes. It will shed light on elucidating the molecular mechanisms underlying the resistance of $B P H 41$ and the genetic effects between $B P H 41$ and other minor QTLs.

\section{Declarations}

Funding: This research was funded by National Natural Science Foundation of China (No.31860416, No.31460387); Science and Technology Project of Guangxi(No.GuikeAB16380093); Key projects of Guangxi Natural Science Foundation of china (Guangdong-Guangxi Joint Fund Project, No. 2021GXNSFDA075013)

Conflicts of Interest: The authors declare no conflict of interest.

Authors' contributions: RL and JL designed and supervised the study, $X W, Y H, Y Z, B D, B W, X G, Y Q, Y F, F L, B Q$ performed the phenotypic data collection. $\mathrm{XW}$ analyzed the data and drafted the manuscript, $\mathrm{RL}, \mathrm{JL}$ and $Y Z$ revised and finalized the manuscript. All the authors read and approved the manuscript. 


\section{References}

Abe, A., Kosugi, S., Yoshida, K., Natsume, S., Takagi, H., Kanzaki, H., Matsumura, H., Yoshida, K., Mitsuoka, C., Tamiru, M., Innan, H., Cano, L., Kamoun, S., \& Terauchi, R. (2012). Genome sequencing reveals agronomically important loci in rice using MutMap. Nature biotechnology, 30(2), 174-178. https://doi.org/10.1038/nbt.2095

Akanksha, S., Jhansi Lakshmi, V., Singh, A. K., Deepthi, Y., Chirutkar, P. M., Ramdeen, Balakrishnan, D., Sarla, N., Mangrauthia, S. K., \& Ram, T. (2019). Genetics of novel brown planthopper Nilaparvata lugens (Stål) resistance genes in derived introgression lines from the interspecific cross 0 . sativa var. Swarna $\times$ O. nivara. Journal of genetics, 98,113 .

Backus, E. A., Serrano, M. S., \& Ranger, C. M. (2005). Mechanisms of hopperburn: an overview of insect taxonomy, behavior, and physiology. Annual review of entomology, 50, 125-151.

https://doi.org/10.1146/annurev.ento.49.061802.123310

Bisht, D. S., Bhatia, V., \& Bhattacharya, R. (2019). Improving plant-resistance to insect-pests and pathogens: The new opportunities through targeted genome editing. Seminars in cell \& developmental biology, 96, 65-76. https://doi.org/10.1016/j.semcdb.2019.04.008

Du, B., Zhang, W., Liu, B., Hu, J., Wei, Z., Shi, Z., He, R., Zhu, L., Chen, R., Han, B., \& He, G. (2009). Identification and characterization of Bph14, a gene conferring resistance to brown planthopper in rice. Proceedings of the National Academy of Sciences of the United States of America, 106(52), 2216322168. https://doi.org/10.1073/pnas.0912139106

Fekih, R., Takagi, H., Tamiru, M., Abe, A., Natsume, S., Yaegashi, H., Sharma, S., Sharma, S., Kanzaki, H., Matsumura, H., Saitoh, H., Mitsuoka, C., Utsushi, H., Uemura, A., Kanzaki, E., Kosugi, S., Yoshida, K., Cano, L., Kamoun, S., \& Terauchi, R. (2013). MutMap+: genetic mapping and mutant identification without crossing in rice. PloS one, 8(7), e68529. https://doi.org/10.1371/journal.pone.0068529

Frisvold G. B. (2019). How low can you go? Estimating impacts of reduced pesticide use. Pest management science, 75(5), 1223-1233. https://doi.org/10.1002/ps.5249

Fujita D, Kohli A, Horgan FG (2013) Rice resistance to planthoppers and leafhoppers. Crit Rev Plant Sci 32(3):162-191. https://doi.org/10.1080/07352689.2012.735986

Guo, J., Xu, C., Wu, D., Zhao, Y., Qiu, Y., Wang, X., Ouyang, Y., Cai, B., Liu, X., Jing, S., Shangguan, X., Wang, H., Ma, Y., Hu, L., Wu, Y., Shi, S., Wang, W., Zhu, L., Xu, X., Chen, R.,Du, B.,He, G. (2018). Bph6 encodes an exocyst-localized protein and confers broad resistance to planthoppers in rice. Nature genetics, $50(2)$, 297-306.

Gupta, P. K., Kulwal, P. L., \& Jaiswal, V. (2019). Association mapping in plants in the post-GWAS genomics era. Advances in genetics, 104, 75-154. https://doi.org/10.1016/bs.adgen.2018.12.001 
Han, Y., Lv, P., Hou, S., Li, S., Ji, G., Ma, X., Du, R., \& Liu, G. (2015). Combining Next Generation Sequencing with Bulked Segregant Analysis to Fine Map a Stem Moisture Locus in Sorghum (Sorghum bicolor L. Moench). PloS one, 10(5), e0127065. https://doi.org/10.1371/journal.pone.0127065

He J, Liu YQ, Liu YL, Jiang L, Wu H, Kang HY, Liu SJ, Chen LM, Liu X, Cheng XN, Wan JM (2013) Highresolution mapping of brown planthopper $(\mathrm{BPH})$ resistance gene $\mathrm{Bph} 27(\mathrm{t})$ in rice (Oryza sativa L.). Mol Breeding 31:549-557

Hill, J. T., Demarest, B. L., Bisgrove, B. W., Gorsi, B., Su, Y. C., \& Yost, H. J. (2013). MMAPPR: mutation mapping analysis pipeline for pooled RNA-seq. Genome research, 23(4), 687-697.

https://doi.org/10.1101/gr.146936.112

Hirabayashi H, Angeles ER, Kaji R, Ogawa T, Brar DS, Khush GS. (1998). Identification of brown planthopper resistance gene derived from o. officinalis using molecular markers in rice. Breed Sci ,48(Suppl):82 (in Japanese)

Hou L Y, Peng S T, Xing-Hua W, et al. (2011) Genetic Analysis and Preliminary Mapping of Two Recessive Resistance Genes to Brown Planthopper, Nilaparvata lugens Stl in Rice[J]. Rice Science, 18(003):238-242.

Hu, J. , Xiao, C. , Cheng, M. , Gao, G. , Zhang, Q. , \& He, Y. . (2015). Fine mapping and pyramiding of brown planthopper resistance genes qbph3 and qbph4 in an introgression line from wild rice o. officinalis. Molecular Breeding, 35(1), 3.

Hu, J., Chang, X., Zou, L., Tang, W., \& Wu, W. (2018). Identification and fine mapping of Bph33, a new brown planthopper resistance gene in rice (Oryza sativa L.). Rice (New York, N.Y.), 11(1), 55. https://doi.org/10.1186/s12284-018-0249-7

Hu, J., Xiao, C., \& He, Y. (2016). Recent progress on the genetics and molecular breeding of brown planthopper resistance in rice. Rice (New York, N.Y.), 9(1), 30. https://doi.org/10.1186/s12284-016-0099-0

Hu, J., Xiao, C., Cheng, M. X., Gao, G. J., Zhang, Q. L., \& He, Y. Q. (2015). A new finely mapped Oryza australiensis-derived QTL in rice confers resistance to brown planthopper. Gene, 561(1), 132-137. https://doi.org/10.1016/j.gene.2015.02.026

Huang, D., Qiu, Y., Zhang, Y., Huang, F., Meng, J., Wei, S., Li, R., \& Chen, B. (2013). Fine mapping and characterization of $\mathrm{BPH} 27$, a brown planthopper resistance gene from wild rice (Oryza rufipogon Griff.). TAG. Theoretical and applied genetics. Theoretische und angewandte Genetik, 126(1), 219229.https://doi.org/10.1007/s00122-012-1975-7

IRRI (1988) Standard evaluation system for rice resistance to brown planthopper. International Rice Research Institute, Manila 
Ishii T, Brar DS, Multani DS, Khush GS. (1994) Molecular tagging of genes for brown planthopper resistance and earliness introgressed from Oryza australiensis into cultivated rice, 0 . sativa. Genome. Apr;37(2):217-21. doi: 10.1139/g94-030. PMID: 18470071.

Ji H, Kim SR, Kim YH, Suh JP, Park HM, Sreenivasulu N, Misra G, Kim SM, Hechanova SL, Kim H, Lee GS, Yoon UH, Kim TH, Lim H, Suh SC, Yang J, An G, Jena KK. (2016) Map-based Cloning and Characterization of the BPH18 Gene from Wild Rice Conferring Resistance to Brown Planthopper (BPH) Insect Pest. Sci Rep. Sep 29;6:34376.

Jia, Q., Wang, J., Zhu, J., Hua, W., Shang, Y., Yang, J., \& Liang, Z. (2017). Toward Identification of Black Lemma and Pericarp Gene Blp1 in Barley Combining Bulked Segregant Analysis and Specific-Locus Amplified Fragment Sequencing. Frontiers in plant science, 8, 1414.

https://doi.org/10.3389/fpls.2017.01414

Kaminski, K. P., Kørup, K., Andersen, M. N., Sønderkær, M., Andersen, M. S., Kirk, H. G., et al. (2016) Next generation sequencing bulk segregant analysis of potato support that differential flux into the cholesterol and stigmasterol metabolite pools is important for steroidal glycoalkaloid content. Potato Res. 59, 8197.

Kamolsukyeunyong, W., Ruengphayak, S., Chumwong, P., Kusumawati, L., Chaichoompu, E., Jamboonsri, W., Saensuk, C., Phoonsiri, K., Toojinda, T., \& Vanavichit, A. (2019). Identification of spontaneous mutation for broad-spectrum brown planthopper resistance in a large, long-term fast neutron mutagenized rice population. Rice (New York, N.Y.), 12(1), 16. https://doi.org/10.1186/s12284-019-0274-1

Klein, H., Xiao, Y., Conklin, P. A., Govindarajulu, R., Kelly, J. A., Scanlon, M. J., Whipple, C. J., \& Bartlett, M. (2018). Bulked-Segregant Analysis Coupled to Whole Genome Sequencing (BSA-Seq) for Rapid Gene Cloning in Maize. G3 (Bethesda, Md.), 8(11), 3583-3592. https://doi.org/10.1534/g3.118.200499

Kumar, K., Sarao, P. S., Bhatia, D., Neelam, K., Kaur, A., Mangat, G. S., Brar, D. S., \& Singh, K. (2018). Highresolution genetic mapping of a novel brown planthopper resistance locus, Bph34 in Oryza sativa L. X Oryza nivara (Sharma \& Shastry) derived interspecific F2 population. TAG. Theoretical and applied genetics. Theoretische und angewandte Genetik, 131(5), 1163-1171. https://doi.org/10.1007/s00122018-3069-7

Li, H., \& Durbin, R. (2009). Fast and accurate short read alignment with Burrows-Wheeler transform. Bioinformatics (Oxford, England), 25(14), 1754-1760. https://doi.org/10.1093/bioinformatics/btp324

Li, Z., Xue, Y., Zhou, H., Li, Y., Usman, B., Jiao, X., Wang, X., Liu, F., Qin, B., Li, R., \& Qiu, Y. (2019). Highresolution mapping and breeding application of a novel brown planthopper resistance gene derived from wild rice (Oryza. rufipogon Griff). Rice (New York, N.Y.), 12(1), 41. https://doi.org/10.1186/s12284-0190289-7 
Liang, T., Chi, W., Huang, L., Qu, M., Zhang, S., Chen, Z. Q., Chen, Z. J., Tian, D., Gui, Y., Chen, X., Wang, Z., Tang, W., \& Chen, S. (2020). Bulked Segregant Analysis Coupled with Whole-Genome Sequencing (BSASeq) Mapping Identifies a Novel pi21 Haplotype Conferring Basal Resistance to Rice Blast Disease. International journal of molecular sciences, 21(6), 2162. https://doi.org/10.3390/ijms21062162

Liu GQ, Yan H, Fu Q, Qian Q, Zhang ZT, Zhai WX, Zhu LH ( 2001) Mapping of a new gene for brown planthopper resistance in cultivated rice introgressed from Oryza eichingeri. Chin Sci Bull. 46:738-742

Liu Y, Wu H, Chen H, Liu Y, He J, Kang H, Sun Z, Pan G, Wang Q, Hu J, Zhou F, Zhou K, Zheng X, Ren Y, Chen L, Wang Y, Zhao Z, Lin Q, Wu F, Zhang X, Guo X, Cheng X, Jiang L, Wu C, Wang H, Wan J. (2015). A gene cluster encoding lectin receptor kinases confers broad-spectrum and durable insect resistance in rice. Nature biotechnology, 33(3), 301-305. https://doi.org/10.1038/nbt.3069

Lv, W., Du, B., Shangguan, X., Zhao, Y., Pan, Y., Zhu, L., He, Y., \& He, G. (2014). BAC and RNA sequencing reveal the brown planthopper resistance gene BPH15 in a recombination cold spot that mediates a unique defense mechanism. BMC genomics, 15(1), 674. https://doi.org/10.1186/1471-2164-15-674

McKenna, A., Hanna, M., Banks, E., Sivachenko, A., Cibulskis, K., Kernytsky, A., Garimella, K., Altshuler, D., Gabriel, S., Daly, M., \& DePristo, M. A. (2010). The Genome Analysis Toolkit: a MapReduce framework for analyzing next-generation DNA sequencing data. Genome research, 20(9), 1297-1303.

Meng L, Li H, Zhang L, Wang J. (2015) QTL IciMapping: integrated software for genetic linkage map construction and quantitative trait locus mapping in biparental populations. The Crop Journal 3:269-283

Mizuno, H., Katagiri, S., Kanamori, H., Mukai, Y., Sasaki, T., Matsumoto, T., \& Wu, J. (2020). Evolutionary dynamics and impacts of chromosome regions carrying R-gene clusters in rice. Scientific reports, 10(1), 872. https://doi.org/10.1038/s41598-020-57729-w

Mohanty, S.K., Panda, R.S., Mohapatra, S.L. Nanda, A., Behera, L., Jena,M., Sahu, R.K., Sahu, S.C., Mohapatra, T. (2017) Identification of novel quantitative trait loci associated with brown planthopper resistance in the rice landrace Salkathi. Euphytica 213, 38. https://doi.org/10.1007/s10681-017-1835-2

Murray, M. G., \& Thompson, W. F. (1980). Rapid isolation of high molecular weight plant DNA. Nucleic acids research, 8(19), 4321-4325. https://doi.org/10.1093/nar/8.19.4321

Navarro-Escalante, L., Zhao, C., Shukle, R., \& Stuart, J. (2020). BSA-Seq Discovery and Functional Analysis of Candidate Hessian Fly (Mayetiola destructor) Avirulence Genes. Frontiers in plant science, 11, 956. https://doi.org/10.3389/fpls.2020.00956

Nguyen, K. L., Grondin, A., Courtois, B., \& Gantet, P. (2019). Next-Generation Sequencing Accelerates Crop Gene Discovery. Trends in plant science, 24(3), 263-274. https://doi.org/10.1016/j.tplants.2018.11.008

Normile D. (2008). Agricultural research. Reinventing rice to feed the world. Science (New York, N.Y.), 321(5887), 330-333. https://doi.org/10.1126/science.321.5887.330 
Pandey, M. K., Khan, A. W., Singh, V. K., Vishwakarma, M. K., Shasidhar, Y., Kumar, V., Garg, V., Bhat, R. S., Chitikineni, A., Janila, P., Guo, B., \& Varshney, R. K. (2017). QTL-seq approach identified genomic regions and diagnostic markers for rust and late leaf spot resistance in groundnut (Arachis hypogaea L.). Plant biotechnology journal, 15(8), 927-941. https://doi.org/10.1111/pbi.12686

Qiu, Y., Guo, J., Jing, S., Zhu, L., \& He, G. (2012). Development and characterization of japonica rice lines carrying the brown planthopper-resistance genes BPH12 and BPH6. TAG. Theoretical and applied genetics. Theoretische und angewandte Genetik, 124(3), 485-494. https://doi.org/10.1007/s00122-011$1722-5$

Rahman, M. L., Jiang, W., Chu, S. H., Qiao, Y., Ham, T. H., Woo, M. O., Lee, J., Khanam, M. S., Chin, J. H., Jeung, J. U., Brar, D. S., Jena, K. K., \& Koh, H. J. (2009). High-resolution mapping of two rice brown planthopper resistance genes, Bph20(t) and Bph21(t), originating from Oryza minuta. TAG. Theoretical and applied genetics. Theoretische und angewandte Genetik, 119(7), 1237-1246.

https://doi.org/10.1007/s00122-009-1125-z

Renganayaki K, Feitz AK, Sadasivam S, Pammi S, Harrington SE, McCouch SR, Kumar SM, Reddy AS (2002)Mapping and progress toward map-based cloning of brown planthopper biotype-4 resistance gene introgressed from Oryza officinalis into cultivated rice, O. sativa. Crop Sci ,42:2112-2117

Sharma PN, Ketipearachchi Y, Murata K, Torii A, Takumi S, Mori N, Nakamura C. (2003)RFLP/AFLP mapping of a brown planthopper (Nilaparvata lugens Stål) resistance gene Bph1 in rice. Euphytica, 129(1):109-117

Singh, V. K., Khan, A. W., Saxena, R. K., Sinha, P., Kale, S. M., Parupalli, S., Kumar, V., Chitikineni, A., Vechalapu, S., Sameer Kumar, C. V., Sharma, M., Ghanta, A., Yamini, K. N., Muniswamy, S., \& Varshney, R. K. (2017). Indel-seq: a fast-forward genetics approach for identification of trait-associated putative candidate genomic regions and its application in pigeonpea (Cajanus cajan). Plant biotechnology journal, 15(7), 906-914. https://doi.org/10.1111/pbi.12685

Song, J., Li, Z., Liu, Z., Guo, Y., \& Qiu, L. J. (2017). Next-Generation Sequencing from Bulked-Segregant Analysis Accelerates the Simultaneous Identification of Two Qualitative Genes in Soybean. Frontiers in plant science, 8, 919. https://doi.org/10.3389/fpls.2017.00919

Sun L, Su C, Wang C, Zhai HQ, Wan JM.(2005) Mapping of a major resistance gene to brown planthopper in the rice cultivar Rathu Heenati. Breed Sci .55:391-396

Sun, J., Yang, L., Wang, J., Liu, H., Zheng, H., Xie, D., Zhang, M., Feng, M., Jia, Y., Zhao, H., \& Zou, D. (2018). Identification of a cold-tolerant locus in rice (Oryza sativa L.) using bulked segregant analysis with a nextgeneration sequencing strategy. Rice (New York, N.Y.), 11(1), 24. https://doi.org/10.1186/s12284-0180218-1 
Tao, Y., Niu, Y., Wang, Y., Chen, T., Naveed, S. A., Zhang, J., Xu, J., \& Li, Z. (2018). Genome-wide association mapping of aluminum toxicity tolerance and fine mapping of a candidate gene for Nrat1 in rice. PloS one, 13(6), e0198589. https://doi.org/10.1371/journal.pone.0198589

Wang H, Shi S, Guo Q, Nie L, Du B, Chen R, Zhu L, He G (2018) Highresolution mapping of a gene conferring strong antibiosis to brown planthopper and developing resistant near-isogenic lines in 9311 background. Mol Breed ,38:107

Wang, Y., Cao, L., Zhang, Y., Cao, C., Liu, F., Huang, F., Qiu, Y., Li, R., \& Lou, X. (2015). Map-based cloning and characterization of $\mathrm{BPH} 29$, a B3 domain-containing recessive gene conferring brown planthopper resistance in rice. Journal of experimental botany, 66(19), 6035-6045.

https://doi.org/10.1093/jxb/erv318

Wu, S., Qiu, J., \& Gao, Q. (2019). QTL-BSA: A Bulked Segregant Analysis and Visualization Pipeline for QTL-seq. Interdisciplinary sciences, computational life sciences, 11(4), 730-737.

https://doi.org/10.1007/s12539-019-00344-9

Yang L, Li R B, Li Y R, Huang F K, Chen Y Z, Huang S S, Huang L F, Liu C, Ma Z F, Huang D H, Jiang J J. (2012) Genetic mapping of bph20(t) and bph21(t) loci conferring brown planthopper resistance to Nilaparvata lugens Stål in rice (Oryza sativa L.). Euphytica, 183: 161-171.

ZHANG Yuexiong, QIN Gang,MA Qianqian, WEI Minyi, YANG Xinghai, MA Zengfeng, LIANG Haifu, LIU Chi, LI Zhenjing, LIU Fang, HUANG Dahui, LI Rongbai.(2020)Identification of Major Locus Bph35 Resistance to Brown Planthopper in Rice[J].Rice Science,27(03):237-247.

Zheng, X., Zhu, L., \& He, G. (2020). Genetic and molecular understanding of host rice resistance and Nilaparvata lugens adaptation. Current opinion in insect science, 45, 14-20. Advance online publication. https://doi.org/10.1016/j.cois.2020.11.005

\section{Figures}
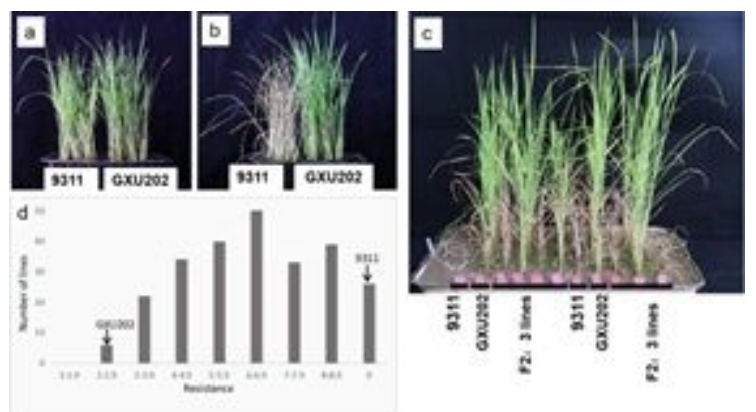

Figure 1 
Resistance phenotypes of GXU202, 9311 and their F2 population to BPH at seedling stage using the seedling bulk test. (a) Phenotypes of GXU202 and 9311 before infested with BPH; (b) Phenotypes of GXU202 and 9311 after infested with BPH; (c) Phenotypes of representative resistant and susceptible F2:3 lines derived from the cross GXU202/9311 after BPH infestation;
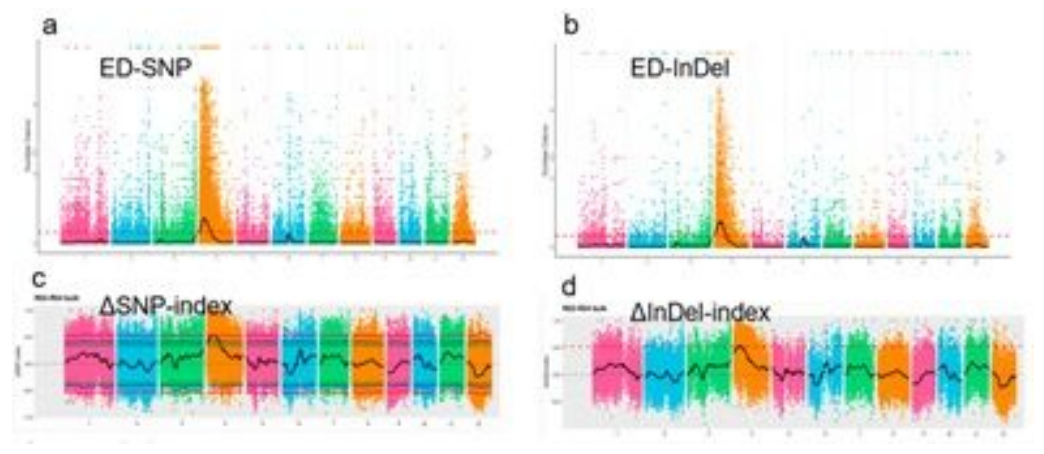

\section{Figure 2}

The profiling of SNPs and small InDels identified by BSA-seq. $(a, b)$ Distribution of Euclidean Distance (ED) correlation value of SNPs (a) and small InDels (b) across all chromosomes. The X-axis represents the chromosome name, the colored dots represent the ED value of each SNP and InDel site, the black line represented the fitted ED value, and the red dotted line represents the significance association threshold. Higher value suggests a better association effect of the corresponding loci. The value of non-targeted loci tends to be 0. (c, d) Distribution of $\Delta$ (SNP-index) values (c) and $\Delta$ (InDel-index) (d) plot across all chromosomes. The X-axis indicates the chromosome name, and the Y-axis indicates the $\Delta(\mathrm{SNP} / \mathrm{InDel}$ index) values. The colored dots represent the calculated $\Delta$ (SNP-index)/ $\Delta$ (InDel-index) value, and the black line represents the fitted $\Delta($ SNP-index $) / \Delta$ (InDel-index) value. The green line, blue line, and red line represents the threshold line with a confidence level of $0.90,0.95$, and 0.99 , respectively. The stronger the asso-ciation between certain SNP and trait, the closer of the $\Delta$ (SNP index) to 1.

(a)

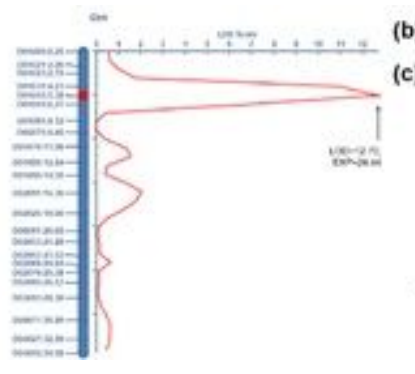

(b)

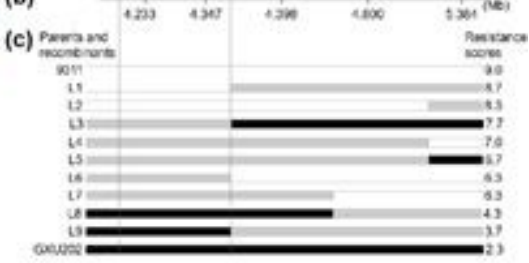

Figure 3

Genetic mapping of the BPH41 gene. (a) Genetic map of chromosome 4 and QTL mapping of the BPH resistance in rice. Molecular linkage map and chromosomal locations (unit: $\mathrm{CM}$ ) of SSR markers on chromosome 4 are presented along the Y-axis on the left. QTL mapping curve of logarithm odds (LOD) scores are presented on the abscissa-axis. The red bar rep-resents the QTL locus with the LOD value above 3. EXP, phenotypic variance explained by the locus. (b) Physical map of marker interval D01031D01045 harboring BPH41. (c) Graphical genotypes and resistance phenotypes of the recombinants. The 
black, white and gray bars denote the marker genotypes of GXU202 homozygotes, 9311 homozygotes and their het-erozygotes, respectively. L1-L9 were the selected recombinants from the BC2F2 population.

\section{Supplementary Files}

This is a list of supplementary files associated with this preprint. Click to download.

- Figures1.jpg

- Figures2.jpg

- Figures3.jpg

- Figures4.jpg

- FigureS5.jpg 